\title{
Resistencia a los antimicrobianos en aislados de Escherichia coli y Enterobacter spp. aisladas de pinzones terrestres de la isla Santa Cruz.
}

López Fiallos, Vanessa Fernanda ${ }^{1}$; Baquero Cárdenas, María Inés ${ }^{1 *}$; Cruz Bedón, Sandra Pía Marilyn²; Duque Suárez, Viviana Margarita²; Vélez Pinela, Edgar Alberto²; Vinueza Burgos, Christian Vinicio' ${ }^{1}$ y Giacoboni, Gabriela Isabel ${ }^{3}$

\author{
${ }^{1}$ Universidad Central del Ecuador, Quito, Ecuador. \\ 2 Agencia de Regulación y Control para la Bioseguridad y Cuarentena de Galápagos, Santa Cruz, Ecuador. \\ ${ }^{3}$ Universidad Nacional de la Plata, La Plata, Argentina. \\ e-mail: mibaquero@uce.edu.ec
}

Palabras clave: Centinela, pinzones, resistencia antimicrobiana.

INTRODUCCIÓN: El incremento de resistencia antimicrobiana es un desafío para la salud global. [1] La vida silvestre juega un papel importante para entender la dinámica de resistencia. [2] Se ha reportado resistencia antimicrobiana en aves encontradas en hábitats remotos. [3] Existe la posibilidad de estudiar aves silvestres como centinelas para vigilar la propagación de cepas resistentes. [2] Los pinzones están expuestos a factores para adquirir y esparcir cepas resistentes; por lo que resultan de interés dentro del campo.

OBJETIV0: Determinar resistencia fenotípica en aislados de Escherichia coli y Enterobacter spp. procedentes de pinzones terrestres de Santa Cruz.

MATERIALES Y MÉTOdOS: Se colectaron hisopados cloacales de tres zonas dentro de la isla: agropecuaria, protegida y urbana; para observar si existen los mismos patrones de resistencia. Se utilizaron redes de neblina para capturar los individuos. Las muestras se conservaron con BHI y glicerol hasta su procesamiento. La fenotipificación se realizó por el método de difusión en disco KirbyBauer, probando puntos de corte establecidos por el CLSI. Los antibióticos usados fueron: gentamicina, cloranfenicol, cefotaxima, cefepima, cefoxitina, ampicilina, ciprofloxacina, trimetoprima sulfametoxazol y tetraciclina.

RESULTADOS: De los 61 hisopados, $50 \%$ de los aislados corresponden a Enterobacter spp. y $14 \%$ a Escherichia coli. En la zona urbana, se encontraron altos índices de resistencia a todos los antibióticos probados en el caso de Enterobacter spp., excepto para la trimetoprima sulfametoxazol y cloranfenicol, donde no se presentó resistencia; para E. coli solo se encontró resistencia a ampicilina y cefoxitina. Por otro lado, en la zona agropecuaria se encontró resistencia a todos los antibióticos en Enterobacter spp. a diferencia de E. coli que solo presentó sensibilidad a trimetoprima sulfametoxazol. En la zona protegida los índices de resistencia fueron menores, se presentó sensibilidad a la tetraciclina y cloranfenicol en el caso de Enterobacter spp.; mientras que, E. coli solo presentó resistencia para la ampicilina y cloranfenicol. Además, de los 58 antibiogramas, el 36,07\% presentó cepas multirresistentes, en su mayoría de la zona agropecuaria.

CONCLUSIONES: La presión antropogénica puede contribuir con la presencia de cepas resistentes en especies de vida silvestre; sin embargo, más estudios deben realizarse para establecer bacterias centinelas.

\section{REFERENCIAS:}

[1] Guenther S, Grobbel M, Lübke-Becker A, Goedecke A, Friedrich ND, Wieler L H, Ewers C. Antimicrobial resistance profiles of Escherichia coli from common European wild bird species. Vet Microbiol. 2009; 219-225. 
[2] Bonnedahl J, Järhult JD. Antibiotic resistance in wild birds. Upsala J Med Sci. 2014; 113-116.

[3] Ramey AM, Hernández J, Tylor V, UherKoch BD, Schmutz JA, Atterby C, Bonnedahl J. AntibioticResistant Escherichia coli in Migratory Birds Inhabiting Remote Alaska. Ecohealth. 2017 